\title{
Changes over time in family members of adults with mild traumatic brain injury
}

Kelly Jones, Alice Theadom, Philip Prah, Nicola Starkey, Suzanne Barker-Collo, Shanthi Ameratunga, and Valery L. Feigin, for the BIONIC Study*

Kelly M. Jones, PhD, National Institute for Stroke and Applied Neurosciences, School of Public Health \& Psychosocial Studies, Faculty of Health and Environmental Studies, Auckland University of Technology, AUT North campus, Private Bag 92006, Auckland

1142, New Zealand. Telephone: +64 9921 9999; Email: kelly.jones@aut.ac.nz

Alice Theadom, PhD, National Institute for Stroke and Applied Neurosciences, School of Public Health \& Psychosocial Studies, Faculty of Health and Environmental Studies, Auckland University of Technology, AUT North Campus, AR417, Private Bag 92006, Auckland 1142, New Zealand. Telephone: +64 9921 9999; Email:

alice.theadom@aut.ac.nz

Philip Prah, MSc, National Institute for Stroke and Applied Neurosciences, School of Public Health \& Psychosocial Studies, Faculty of Health and Environmental Studies, Auckland University of Technology, AUT North campus, Private Bag 92006, Auckland 1142, New Zealand. Telephone: +64 9921 9999; Email: philip.prah@ aut.ac.nz

Nicola J. Starkey, PhD, School of Psychology, Faculty of Arts \& Social Sciences, The University of Waikato, Private Bag 3105, Hamilton, New Zealand. Telephone: +64 7837 9230; Email: nstarkey@ waikato.ac.nz

Suzanne Barker-Collo, PhD, School of Psychology, Tamaki Campus, The University of Auckland, Private Bag 92019, Auckland, New Zealand.

Telephone: +64 93937599 ext 88517; Email: $\underline{\text { s.barker-collo@auckland.ac.nz }}$ 
Shanthi Ameratunga, PhD, School of Population Health, Faculty of Medical \& Health

Sciences, The University of Auckland, Private Bag 92019, Auckland, New Zealand. Telephone: +64 9923 6354; Email: s.ameratunga@auckland.ac.nz

Valery L Feigin, PhD, National Institute for Stroke and Applied Neurosciences, School of Public Health \& Psychosocial Studies, Faculty of Health and Environmental Studies, Auckland University of Technology, AUT North campus, Private Bag 92006, Auckland 1142, New Zealand.

Telephone: +649921 9166; Email: valery.feigin@aut.ac.nz *BIONIC Study Group members listed below

Address correspondence to: Dr Kelly M Jones, PhD, National Institute for Stroke and Applied Neurosciences, School of Public Health \& Psychosocial Studies, Faculty of Health and Environmental Studies, Auckland University of Technology, AUT North campus, Private Bag 92006, Auckland 1142, New Zealand. Telephone: +64 9921 9166; Email: kelly.jones@aut.ac.nz 


\section{ABSTRACT}

\section{Objective}

The impact of traumatic brain injury (TBI) extends beyond the person who was injured. Family caregivers of adults with moderate to severe TBI frequently report increased burden, stress, and depression. Few studies have examined the well-being of family members in the mild TBI population despite the latter representing up to $95 \%$ of all TBIs.

\section{Methods}

Five areas of well-being were examined in 99 family members (including parents, partners, siblings, other relatives, adult children, friends or neighbours) of adults (aged $\geq 16$ years) with mild TBI. At 6- and 12-months post-injury, family members completed the Bakas Caregiver Outcomes Scale, Short Form-36 Health Survey, EQ-5D-3L, Hospital Anxiety and Depression Scale, and the Pittsburgh Sleep Quality Index. Outcomes and change over time, and associated factors were examined.

\section{Results}

At 6-months, group mean scores for health-related quality of life for mental and physical components, and overall health status were similar to the New Zealand (NZ) population. Mean scores for sleep, anxiety, and depression were below clinically significant thresholds. From 6- to 12-months, there were significant improvements in Bakas Caregiver Outcomes Scale scores by 2.61 (95\%CI: 0.72-4.49), health-related quality of life (mental component) and EQ-5D-3L overall health $(\mathrm{p}=0.01)$. Minimally clinically important differences were observed in overall health, anxiety, health-related quality of life and depression at 12-months. Female family members reported significant improvements in physical health over time and more positive life changes were reported by those caring for males with TBI. 


\section{Conclusions}

Findings suggest diminished burden over time for family members of adults with mild TBI.

Key words: mild traumatic brain injury; family members; outcomes; sleep; health. 


\section{INTRODUCTION}

Traumatic brain injury (TBI) affects at least 50 million people worldwide each year, and is a major cause of disability across all ages in developing and developed countries (Maas et al., 2017). Clear differences have been established between outcomes and TBI severity. Meta-regressions exploring associations between injury severity and intellectual impairments reveal significant linear associations between mild, moderate and severe TBI and poorer full scale IQ among adults in the acute and subacute phases of recovery (Königs, Engenhorst, \& Oosterlaan, 2016). Polinder and colleagues undertok a prospective cohort study of $996($ mild $n=797$, moderate $n=50$, severe $n=$ 149) adult TBI patients (median age 44 years, $63 \%$ male). Results showed that moderate to severe TBI was associated with significantly poorer outcomes than mild TBI on Short-Form (SF-36) Health Survey domains of physical functioning, role physical, social functioning and role emotional $(F=8.5-20.4$, df $=2$, all $\mathrm{p}<0.001)$. At 1 -year follow-up only those with mild TBI were comparable to Dutch population norms on all scales of the SF-36 (Scholten et al., 2015).

While such findings reveal less impact of mild than more severe TBI, it is increasingly acknowledged that the 'mild' label is a misnomer in some cases (Maas et al., 2017). Mild TBI has been associated with increased risks for affective disorders (Delmonico, Theodore, Sandel, Armstrong, \& Camica, 2017), suicide (Madsen, Erlangsen, \& Orlovska, 2018), cognitive deficits (McInnes, Friesen, MacKenzie, Westwood, \& Boe, 2017), and reduced community participation (Carroll et al., 2014; Theadom et al., 2018) in the longer term. At 1-year post-injury, $41 \%$ of adults may have clinically significant sleep difficulties, with $21 \%$ at a level of insomnia (Theadom et al., 2015). Furthermore, up to $95 \%$ of all TBI will be mild in severity (Feigin et al., 2013) making major 
contributions to non-fatal outcomes (Te Ao et al., 2015). Greater prevalence of mild TBI and the breadth of potentially adverse outcomes may affect spouses, neighbours, friends, extended family, or other relatives (herein broadly referred to as 'family members') who take on additional roles to support the injured person and family unit. Family members may not adjust well to required changes (Marsh, Kersel, Havill, \& Sleigh, 2002) and disruptions to their daily routines and social, vocational, and recreational activities (Nonterah et al., 2013). A systematic review of 62 studies of predominantly moderate to severe TBI samples revealed widespread evidence of carer burden, being the physical, psychological, emotional, social, and financial consequences of caring for someone else (Baker, Barker, Sampson, \& Martin, 2017).

Family members also report increased physical demands in response to supporting someone with moderate to severe TBI, although these demands may reduce over time for some individuals (Marsh et al., 2002). Being a family member of someone with TBI may also be associated with more frequent reporting of health problems, somatic symptoms (e.g. fatigue, headaches, back pain) (Berglund, Lytsy, \& Westerling, 2015; Kreutzer et al., 2009; Rumpold et al., 2015), significantly lower scores for health-related quality of life compared to healthy controls (Arango-Lasprilla et al., 2010), and greater social isolation (Ergh, Hanks, Rapport, \& Coleman, 2003; Hanks, Rapport, \& Vangel, 2007) and reduced participation (Powell et al., 2017). Family members also report concerns for their own emotional adjustment including greater emotional distress, anxiety, depression, caregiver burden (Doser \& Norup, 2016), and unmet family needs (Arango-Lasprilla et al., 2010). Manskow and colleagues' (2014) Norwegian study found that $16 \%$ of caregivers of adults with a severe TBI reported a high level of burden, $34 \%$ reported a medium and 50\% a low level of burden at 1-year after the injury 
(Manskow et al., 2015). Further research revealed that the total caregiver burden increased from between 1- and 2-years following severe TBI. Thirty percent of the family members reported an increased burden, 55\% were stable, and $15 \%$ had a decrease in burden between the two follow-up times (Manskow et al., 2017). Key themes identified by focus group data from 52 informal caregivers of adults with moderate or severe TBI include the burden of additional responsibilities, lack of personal time and time for self-care, feeling that life is interrupted or lost, grief for the person with TBI, and feelings of anger, guilt, anxiety, and sadness (Kratz, Sander, Brickell, Lange, \& Carlozzi, 2017) .

Poor well-being among family members may be associated with poorer recovery of those with TBI, including poor cognitive function, psychosocial well-being, social integration, and functional ability (Sady et al., 2010; Sander, Maestas, Sherer, Malec, \& Nakase-Richardson, 2012; Vangel, Rapport, \& Hanks, 2011). A study of 109 informal caregivers of adults with TBI requiring acute hospitalisation and rehabilitation found associations between increased burden on significant others and poorer neuropsychological functioning in TBI patients at an average of 6 years post-injury (Vangel et al., 2011). The exact underlying mechanisms leading to links between family member well-being and TBI patient outcomes are yet to be determined. Nevertheless, these associations highlight the value of understanding the well-being of family across all levels of TBI severity.

The literature on informal caregiver burden has provided substantial evidence of widespread and significant distress in the context of moderate to severe TBI. While also largely confined to the impact of moderate to severe TBI and not the focus of the 
current study, the impact of mild TBI has been examined to a limited extent among parents of injured children. Minney and colleagues' (2019) Australian study involved semi-structured interviews of 9 parents of children (8-12 years) with mild TBI at 1-2 months post-injury (Minney, Roberts, Mathias, Raftos, \& Kochar, 2019). Findings revealed increases in parent-reported burden in terms of their time and finances due to time off work. Such burden was linked to greater stress, exhaustion and conflict within the family unit. Parents also reported shock, fear, anxiety, and guilt immediately after their child's injury that increased over time due to worries about the long-term impact of TBI and their child's unpredictable emotions and behaviours. As noted in other pediatric TBI studies (Aitken, Mele, \& Barrett, 2004; Armstrong \& Kerns, 2002; Hawley, 2003), unmet needs, including a lack of emotional reassurance during the acute phase, an avoidance of internal feelings to remain "strong" for the family, and recommendations for psychological and counselling support were also reported. However, despite evidence of adverse impacts of mild TBI among parents of injured children, there continue to be substantial gaps in our understanding of how mild TBI impacts the families of adult patients.

There is also little information available about which factors relate to the experiences of family members that can help to predict who will experience better or worse outcomes over time. Female family caregivers of terminally ill cancer patients, for example, commonly report higher levels of stress and burden and lower self-esteem than male caregivers (Schrank et al., 2016). Sex differences are proposed to influence the type and extent of care provided, coping strategy use, and access to informal and formal support to cope with caregiver strain (Hasson-Ohayon et al., 2015; Wadhwa et al., 2013). Female carers tend to spend more hours caring, are more likely than males to 
alter their employment status due to caring needs, and may be less likely to seek support (Mystakidou et al., 2013). Caring for a family member with terminal cancer is clearly far removed from caring for a family member with mild TBI. However, similar associations have been found between the poorer well-being of female family caregivers compared to male caregivers across a range of health populations, including mental illness (Sharma, Chakrabarti, \& Grover, 2016), sclerosis (Qutub, Lacomis, Albert, \& Feingold, 2014), and chronic obstructive pulmonary disease (Nakken et al., 2017). Yet, it is unknown whether similar risks for poor outcomes are evident among those caring for adult family members with mild TBI.

The present study was part of a larger prospective population-based cohort study undertaken in NZ, where it is estimated that 527,000 New Zealanders (13\% of the population) have experienced at least one TBI event at some time in their lives (Te Ao et al., 2015). The prevalence of TBI is $23 \%$ higher in males than it is in females in NZ, adjusting for differences in age distributions. The highest prevalence is among those aged 40-49 years in both males and females, with two-thirds (68\%) of prevalent cases aged 35 years and older. Mild TBI makes the greatest contribution to the non-fatal burden on TBI in NZ (accounting for 56\% of total years lived with disability) (Te Ao et al., 2015).

The study aimed to determine family members' well-being at 6-months post-injury (Aim 1), to examine patterns of change in outcomes in family members from 6- to 12 months post-injury (Aim 2), and to determine if change in outcomes over time can be predicted by specific characteristics of family members, mild TBI patients, and/or injury (Aim 3). 


\section{METHODS}

The study was approved by the Northern Y Health and Disability Ethics Committee of NZ (reference numbers: NTY/09/09/095 and NTY/11/02/016), and the Auckland University of Technology Ethics Committee (reference number: 09/265).

\section{Design}

This was a longitudinal study of family members of adults ( $\geq 16$ years at injury) with mild TBI who were identified as part of the prospective, population-based Brain Injury Incidence and Outcomes In the NZ Community (BIONIC) study. The methodology of the BIONIC study was built upon World Health Organisation (WHO) Injury Surveillance Guidelines (Holder et al., 2001) and details of case ascertainment are reported elsewhere (Theadom et al., 2012). In brief, prospective and retrospective surveillance systems were used to identify all TBI events $(\mathrm{N}=1,369)$ across all ages that occurred in the Hamilton and Waikato districts of NZ over a 1-year period (1 March 2010 through 28 February 2011), including mild non-hospitalised cases often missing from other studies.

Complete case ascertainment was supported by using multiple overlapping sources of information for all the newly hospitalised and non-hospitalised TBI cases (fatal and non-fatal). Hot pursuit methods were used to identify additional cases of TBI through cross-checks of general practitioner databases and hospital admissions. Participants were also identified through searches of claims made through NZ's national accident compensation provider. TBI was defined using WHO criteria as an acute brain injury resulting from mechanical energy to the head from external physical forces (Carroll, 
Cassidy, Holm, Kraus, \& Corondo, 2004), with a Glasgow Coma Score of 13-15 and/or Post Traumatic Amnesia ( $<24$ hours). Operationally, TBI was defined as including the presence of one or more of the following: confusion or disorientation; loss of consciousness; post-traumatic amnesia; and other neurological abnormalities (e.g. seizure) (Carroll et al., 2004). Given that many people do not seek medical attention following a mild TBI, in instances where there was no medical confirmation of diagnosis, details of the accident were obtained and reviewed by a diagnostic team of neurologists, clinicians and neuropsychologists to determine eligibility for the study. Participants were considered eligible if they had described an incident likely to result in a TBI and met at least one of the following criteria: dazed or confused after the accident, loss of consciousness for 30 minutes or less, and/or not being able to remember what happened during the accident.

Family members

Ninety-nine family members met the following 4 criteria: 1 ) they spent a significant proportion of the day with the adult ( $\geq 16$ years at injury) with mild TBI, similar to definitions used in previous research (Bayen et al., 2013); 2) they provided informed consent to take part; 3) they were aged 16 years or over at the time of assessment; and 4) they completed a study assessment at 6- and 12-months to allow the examination of change over time (Figure 1). Family members in the current sample were predominantly female $(73 / 99,74 \%)$, with a mean age of 44 years $(\mathrm{SD}=14.4)($ Table 1$)$. Nearly half (48/99) described themselves as 'partners' of the adult with mild TBI, followed by 'parents' (31\%), and 'other' (20\%). Family members included in the current analysis $(\mathrm{N}=99)$ were compared to those who were excluded $(\mathrm{N}=109)$ due to 
data being available at a single time point. There were no significant between-group differences in age, sex, or type of relationship with the adult with mild TBI.

\section{[INSERT FIGURE I ABOUT HERE]}

\section{[TABLE 1 INSERT HERE]}

\section{Procedure}

Following informed written consent for the outcome component of the BIONIC study, all adults were asked to nominate a family member who might like to take part in the study to help us find out more about the impact of the TBI on the family. Following initial contact, interested family members were sent a study information sheet. One week later, the research team made contact by telephone to answer any questions, discuss the study further, and to seek verbal consent. Those who were interested in taking part were invited to complete in-person assessments at 6- and 12-months postinjury. From the original incidence cohort of 234 adults with a mild TBI who had a family member who consented to take part, 99 (42\%) completed an assessment at 6- and 12-months post-injury (Figure 1). Appointments predominantly took place at the family members' private residence or other suitable location. Informed written consent to take part in the study was sought at the first appointment. Then, family members completed a 60-90 minute assessment capturing demographic information and using standardised questionnaires to assess well-being.

Measures administered to family members

The 15-item Bakas Caregiver Outcomes Scale (BCOS) (Bakas, Champion, Perkins, Farran, \& Williams, 2006) assessed perceived life changes due to being a family member of an adult with mild TBI. This questionnaire covers emotional well-being, 
coping with stress, self-esteem, relationships with friends and family, physical health, time for social activities, future outlook, and relationship with the care recipient. Using a 7-point Likert scale from -3 (changed for the worst) to +3 (changed for the best), item scores are transformed to scores of 1 to 7 and summed. The range for total scores is 15 to 105 . Scores over 60 indicate that life "changed for the best". Scores lower than 60 indicate that life "changed for the worst". A score of 60 indicates "no change". Specifically designed for those assisting people with acquired brain injury, the BCOS has previous use in TBI populations (Powell, Fraser, Brockway, Temkin, \& Bell, 2015). It also has proven internal consistency (Cronbach $\alpha=0.90)$, test-retest reliability (intraclass correlation $=0.68)$, and construct validity (Bakas et al., 2006).

The 36-item medical outcomes Short-Form 36 (SF-36) (Ware \& Sherbourne, 1992) assessed health-related quality of life (QoL). Based on eight subscales, the SF-36 produces two component scales (mental health and physical health) ranging from 0-100. Higher scores indicate better outcomes. Mean total population scores for NZ for the mental health and physical component scores are 50.0 and 50.1, respectively (Ministry of Health, 1999). There are no universally accepted cut-offs to indicate clinically significant problems. The Minimally Clinically Important Difference (MCID) is $\geq 5$ points (Samsa et al., 1999). The SF-36 is commonly used in TBI research (Anderson, Simpson, \& Daher, 2017), with proven psychometric properties (Emanuelson, Andersson Holmkvist, Bjorklund, \& Stalhammar, 2003; Findler, Cantor, Haddad, Gordon, \& Ashman, 2001) and is validated for use in NZ (Scott, Tobias, \& Sarfati, 1999). However, it is important to consider ethnic differences in patterns of response among NZ ethnic subgroups. The factor stucture of the SF-36 does not clearly differentiate physical and health components among older ( $\geq 45$ years) Māori and Māori 
tend to score less well on the SF-36 than NZ Europeans (Scott, Sarfati, Tobias, \& Haslett, 2000).

The visual analogue scale (VAS) of the standardised 3-level EQ-5D health status measure (EQ-5D-3L) (The EuroQol Group, 1990) assessed current health. Each family member was asked to 'mark an $\mathrm{X}$ on the scale to indicate how your health is TODAY' on a $20 \mathrm{~cm}$ vertical VAS. Endpoints range from '100' (best imaginable health state) to '0' (worst imaginable health state). To enable comparisons between the EQ-VAS scores found in the current study and those in the NZ population, it was necessary to adjust study data for potential differences in demographics. This was achieved by aggregating data after age standardisation based on a European population structure (Szende, Janssen, \& Cabases, 2014). The age standardised NZ mean of the EQ-VAS is 81.35 (EuroQol Group, 2004). While no validated cut-off indicating clinical impairment could be found, the established MCID is 10 points (Luo, Johnson, \& Coons, 2010). The psychometric properties of the EQ-5D-3L have been demonstrated in a range of healthy and patient populations (Wang et al., 2012; Zhang, Fu, Wang, \& Du, 2009; Zhou et al., 2012).

Mood was assessed using the self-report Hospital Anxiety and Depression Scale (HADS) (Zigmond \& Snaith, 1983). The HADS contains 7-items relating to anxiety and 7-items relating to depression based on feelings during the past week. Total anxiety and total depression scores range from 0-21. Subscale scores from 11 to 21 indicate moderate to severe anxiety or depression. The established MCID is $\geq 1.5$ points (Bhandari, Jain, Marolda, \& ZuWallack, 2013; Puhan, Frey, Buchi, \& Schunemann, 
2008). The HADS has previously been used with family members of adults with mild to severe TBI (Morris, 2001).

The standardised 19-item Pittsburgh Sleep Quality Index (PSQI) (Buysse, Reynolds, Monk, Berman, \& Kupfer, 1989) subjectively assessed sleep patterns and quality over the past one month. Seven component scores reflect subjective sleep quality, latency, disturbances, efficiency, duration, use of sleeping medication, and daytime dysfunction. Using a '0' (no difficulty) to '3' (severe difficulty) interval scale, all component scores are summed to create a global PSQI score (range 0 to 21). A global PSQI score of 5 or greater indicates poor sleep quality (Omachi, 2011). The MCID in a sample of patients with sleep disturbance is -3 (Hughes et al., 2009). With proven reliability and validity (Mollayeva et al., 2016), the PSQI has been used effectively in longitudinal studies of caregiver outcomes (Lerdal et al., 2016).

Associated variables

Based on research in moderate to severe TBI and chronic medical conditions (Marsh, Kersel, Havill, \& Sleigh, 1998; Martin, 2000), the following variables were examined in relation to changes in key outcomes from 6- to 12-months: family member age, sex, and relationship to patient; patient age at injury, sex, ethnicity (NZ European, Māori, Other); and injury factors (mechanism of injury, prior TBI [yes/no], accidental injury [yes/no], recurrent TBI within 12 months of index injury [yes/no]). With the exception of selfreported prior TBI, injury factors were based on information from medical records. 
Analyses

Descriptive statistics were used to report the characteristics of the sample, using means and standard deviations or frequencies and percentages as appropriate. The results at 6months post-injury were compared to NZ population data to address the first study aim. In relation to the second study aim, we used linear and logistic mixed effect models, with random intercept to take account of the correlation between repeated measurements. The primary outcome was the change (linear model) or odds ratio (logistic model) in each outcome measure from 6- to 12-months. We present unadjusted models for these comparisons. The third research aim was addressed by examining possible interactions between those family member, patient and injury variables shown in Table 1 and change in outcomes over time. Statistical significance was set at $\mathrm{p}<0.05$. All analyses were performed using Stata version 14.0.

\section{RESULTS}

Family member outcomes at 6-months (Aim 1)

As shown in Table 2, family member outcomes at 6-months revealed a mean overall life change score of $58.2(\mathrm{SD}=7.2)$, just below 60 which indicates no change. Group mean scores for health status, QoL for mental and physical components of the SF-36 were consistent with NZ total population mean scores. Mean self-reported ratings of anxiety, and depression were all within normal limits. Mean sleep scores indicated good sleep quality at 6-months.

Changes in outcomes from 6- to 12-months (Aim 2)

As a group and over time, family members reported better well-being (Table 2). From 6- to 12-months, there were significant improvements in BCOS perceived life changes, 
mean scores increased by 2.61 (95\% confidence interval (CI): 0.72-4.49), the mental component of QoL increased by 2.02 (95\% CI: 0.40-3.63), and health status by 3.8 (95\% CI: 0.77-6.83). No significant group level changes were found for QoL physical summary scores (SF-36), anxiety and depression (HADS), and overall sleep quality (PSQI).

Applying MCID thresholds and compared to 6-months, around one quarter of family members reported better QoL physical component scores and less anxiety at 12-months. Over one-third met the MCID threshold for improvements in QoL mental component scores and overall health status (EQ-5D-3L). Reductions in depression (HADS) and improved sleep quality (PSQI) were observed in $13 \%$ and $11 \%$, respectively. There were no significant changes in the proportion of family members meeting established cut-offs for anxiety, depression, or poor sleep at 12-months. The mean age-standardised EQ-5D-3L VAS scores were 79.2 and 81.8 at 6- and 12-months, respectively. These results are similar to the age-standardised scores found in a NZ population survey, 81.3 (EuroQol Group, 2004).

\section{[INSERT TABLE 2 HERE]}

Potential interactions with changes in outcomes from 6- to 12-months (Aim 3) For each outcome, potential interaction effects were examined between change over time and the following factors from Table 1- age and sex of TBI patients and family members, relationship of family member to patient, and patient ethnicity and prior TBI. Table 3 reports estimates from five significant mixed models where overall change over time differs by family member or patient characteristics. A supplementary table reports the results of all potential interactions. 
Family member characteristics: Mean physical health summary scores (SF-36) over time differed by sex with scores increasing by 2.1 (95\% CI: 0.4-3.7) among females, but not males, between 6- and 12-months (Figure 2). A post-hoc analysis of the SF-36 physical health component subscale scores revealed that female family members reported significantly less bodily pain from 6- to 12-months, 7.2 (95\% CI: 1.7-12.7), p $<0.01$. Male family members reported more bodily pain from 6- to 12 -months but the extent of change was not statistically significant, $-5.6(95 \% \mathrm{CI}:-14.8-3.7), \mathrm{p}=0.24$ (Figure 3).

\section{[INSERT FIGURES $2 \& 3$ HERE]}

Patient characteristics: Positive overall life changes (BCOS) from 6- to 12-months were reported by family members of males with mild TBI, 4.33 (95\% CI: 2.02 - 6.65), p $<0.001$. Overall life changes were constant over time for family members of females with mild TBI, -0.30 (95\% CI: $-3.29-2.70), \mathrm{p}=0.84$. Mental health summary scores (SF-36) did improve for family members of NZ European adults with mild TBI, 3.07 (95\% CI: 1.19 - 4.95), p <0.001, but not for family members of Māori adults, -0.73 ($95 \%$ CI: $3.94-2.48), p=0.65$.

Injury characteristics: Family members of adults free from a prior TBI reported less depression (HADS) over time, -0.79 (95\% CI: $-1.34--0.24), \mathrm{p}<0.01$. There were no significant changes in depression for family members of adults with prior TBI, 0.09 (95\% CI: $-0.40-0.59), \mathrm{p}=0.70$.

[INSERT TABLE 3 HERE] 
[INSERT LINK TO SUPPLEMENTARY DIGITAL CONTENT TABLE]

\section{DISCUSSION}

This study examined the well-being of broadly defined family members of adults with mild TBI at 6-months post-injury. We also determined, and identified factors associated with, any changes in outcomes from 6- and 12-months. In relation to our first aim, findings suggest that family members perceived relatively few life changes at 6-months. Family members tended to report similar functioning to adults in the general population in terms of QoL, overall health, mood, and sleep quality at 6-months. With regards to our second aim, as a group, family members generally perceived that life had changed for the better by 12-months with corresponding improvements in QoL mental scores and overall health status. There were no significant changes from 6- to 12-months in QoL physical, anxiety, depression, or sleep. With respect to our third aim, female family members reported significant improvements in physical health, more positive life changes were reported by those caring for males with TBI, mental health improved for family members of TBI patients of NZ European but not Māori ethnicity, and an absence of prior TBI was linked to less depression in family members from 6- to 12months.

In terms of well-being at 6-months, family members reported few overall life changes. To our knowledge, only two other studies in NZ have published data using the BCOS instrument. While focused on older people with stroke, the Auckland Regional Community Stroke Study (ARCOS) (Parag et al., 2008) found mean BCOS scores reflected more changes in life for the worst at 6-months (mean 51.7; SD 12.0) and 18months (mean 52.6; SD 12.0). Similarly, the Prospective Outcomes of Injury Study 
examined carers of injured adults aged 18-64 years after 1-year and found lower carer well-being (mean 53.5; SD 12.6) than in the current study (McAllister, Derrett, Audas, $\&$ Pau, 2012). Further, no significant impacts on mood were observed in the current study. These findings are in contrast to those of another NZ study of 52 primary caregivers of adults with severe TBI (Marsh et al., 1998). Results showed that approximately one-third of caregivers reported clinically significant symptoms of anxiety and depression at 6-months with similar rates at 1-year post-injury (Marsh et al., 1998). Increased physical illness, less time for themselves, and change in employment were reported as causing the most distress for caregivers. Given the less severe nature of mild TBI, it is possible that caregivers do not encounter the same causes of distress as those caring for adults with severe TBI. Therefore, any impact on mood may be relatively minimal. Taken together and reviewed alongside evidence from more severely injured samples, our findings suggest that mild TBI has a lesser impact on family members at 6-months post-injury compared to more severe TBI and acquired brain injury such as stroke.

Observed improvements in terms of statistically and clinically relevant changes from 6to 12-months suggest that the impact of mild TBI on overall life, health and QoL reduces somewhat over time. This pattern of findings is quite the opposite to evidence of increased burden over time among caregivers of family members with moderate to severe TBI. Using the Caregiver Burden Scale in 80 Norwegian families, Manskow and colleagues found that $15 \%$ informal caregivers of adults with severe TBI reported less burden at 1- to 2-years after severe TBI (Manskow et al., 2017). However, overall burden increased significantly from 1- to 2-years post-TBI $(\mathrm{p}=.04)$. Of those that experienced change in caregiver burden over time, 53\% (10/19) and 82\% (14/17) 
reported change in a negative direction in the low- and moderate/high-burden groups at 1-year, respectively. While using the BCOS and examining earlier time points, our findings reveal an opposite pattern of findings. Compared to $60 \%$ at 6 -months, $70 \%$ of family members reported that life had changed for the better by 12-months representing a 10\% improvement. Such reports of better well-being over time may reflect a continuum of improvements from the time of injury.

The mechanisms through which improvements over time occur cannot be determined from the current analysis. However, as suggested by others in relation to severe TBI (Marsh et al., 2002), it may be that perceptions change over time as family members develop and refine coping strategies to manage any on-going impacts of mild TBI. Improvements may also reflect recovery of the patient from TBI and perhaps other related physical injuries or related psychological effects. However, in the current study we found no associations between patient MCIDs in QoL, anxiety or depression over time and changes (MCID or otherwise) in outcomes for family members. Our sample may have been too small to detect changes in outcomes over time. Further, although MCIDs for patients indicate clinical improvement over time, the extent of these changes may have a minimal effect on family member outcomes.

In contrast to reported improvements, no significant changes over time were found for QoL physical, sleep quality, anxiety, or depression. These findings are perhaps to be expected. In the current study, respective mean scores were all within the normal range at 6-months and therefore there was little scope for improvement. A review of 24 studies found that high levels of psychological distress at 12-months or more post-injury are commonly reported by informal caregivers in mild to severe TBI populations 
(Ennis, Rosenbloom, Canzian, \& Topolovec-Vranic, 2013). However, none of the studies in this review focused solely on mild TBI which may at least in part explain the differences in our findings. Lui and colleagues' (2015) cross-sectional descriptive study examined psychological symptoms among 300 significant others of those with mild, moderate, or severe TBI (Liu, Zhu, Liu, \& Guo, 2015). Using the Symptom Checklist90, findings revealed clear linear relationships between more severe TBI and greater psychological symptoms among significant others. Norup and colleagues' study of 94 Danish family members of patients with severe TBI at five time points over the year post-injury found SF-36 HRQoL scores significantly increased over time (Norup et al., 2013). Supportive of evidence of linear associations between TBI severity and greater psychological symptoms, our findings suggest a relatively small impact of mild TBI on the psychological well-being of family members compared to those supporting adults with more severe TBI. In terms of the impact of mild TBI on family member mood over time, our findings align with evidence of stability in anxiety and depression levels across the same time points in severe TBI populations (Marsh et al., 2002). Together, these findings suggest that levels of anxiety and depression present among family members appear to be relatively stable from 6- to 12-months post-injury.

The final aim of our study was to determine if changes in outcomes from 6- to 12months can be predicted by specific family member, patient demographic and/or TBI injury characteristics. Although with a restricted sample size, there was some evidence to indicate an association between improved physical QoL and sex, particularly less bodily pain for family members who were female but not for males. This study may be the first to report a significant interaction between female sex and reports of significantly reduced bodily pain over time within the context of mild TBI. This finding 
adds to evidence, albeit it limited, that there may be sex-differences in some outcomes for those supporting a family member with TBI. Anderson and Simpson (2013) examined the effects of caring for a relative with severe TBI in male $(\mathrm{N}=46)$ versus female $(\mathrm{N}=76)$ caregivers (Anderson \& Simpson, 2013). Male and female caregivers reported similar outcomes, but they differed in their pathways to psychological distress. Unhealthy family functioning had greater impact on the level of psychological distress in male compared to female caregivers. Sex differences in caregiver outcomes have been found in other populations. Females have been found to be at greater risk for stress-related health problems associated with caregiving than their male counterparts in other populations, including cancer (Li \& Loke, 2013) and sclerosis (Qutub et al., 2014). It is important to note that there were only a small number of male family members in our sample and that there were wide variations in their bodily pain scores. Normative SF-36 data for NZ also shows that females tend to rate their health worse than males (Scott et al., 1999) as also found in Australia (Australian Bureau of Statistics, 1997) and the United States (Ware, Snow, Kosinski, \& Gandek, 1993). Further, as concluded by the authors of a recent review of sex differences among caregivers of family members with mental illness (Sharma et al., 2016), the experiences of male caregivers has not been adequately explored. For example, the role of sex on caregiver outcomes may be mediated by effects of kinship status and culture, but these have seldom been considered in research examining sex differences. Further research specifically designed to determine the true nature of sex differences in family caregiving of adults with mild TBI is required.

Similarly, evidence of associations found in the current study between family members' QoL and mood, and patient ethnicity require cautious interpretation. Māori make up on 
average $15 \%$ of NZ's population (Statistics New Zealand, 2017), yet face disproportionate risks for TBI. Compared with people of European origin, Māori have a greater risk of mild TBI (rate ratio $1 \cdot 23,95 \%$ CI 1·08-1·39) (Feigin et al., 2013). Furthermore, disparities in access to supports are well-documented (Jansen, Bacal, \& Crengle, 2008) along with concerns about the appropriateness of using standardised measures based on non-Māori populations. For example, as relevant to the current study, there are concerns about using the two-factor SF-36 structure in Māori. Unlike more European views, traditional views of health among Māori do not recognise the separation of mental and physical health. There is also evidence to suggest that the twodimensional structure of the SF-36 does not replicate well among older Māori (Scott et al., 2000). Alongside measurements concerns, Māori also face elevated risks for factors associated with poor outcomes after TBI, including low SES (Barker-Collo, Wilde, \& Feigin, 2009), incarceration and substance abuse (Barnfied \& Leathem, 1998), exposure to violence (Dannette, Fergusson, \& Boden, 2008), and poor mental health (OakleyBrown, Wells, \& Scott, 2006). Elder (2012) recommends the use of indigenous research methodologies to better understand TBI disparities among Māori and to inform prevention and treatment, including consideration of the concept of 'he tapu te upoko' (the head is sacred) (Elder, 2012). Together, findings from the current study highlight areas for future consideration when endeavouring to identify mechanisms that may be contributing to the well-being of family members in mild TBI populations.

\section{Strengths and Limitations}

Strengths of our study include the high number of family members compared to previous studies, and our examination of a mild TBI sample including recruitment beyond hospitalised patients. A further strength is our examination of predictors of 
change over time rather than predictors of specific outcomes at a single time point. Limitations of our study concern the absence of other predictors found to be important for the well-being of family members in moderate to severe TBI samples. These include consideration of the roles of family functioning (Baker et al., 2017), loneliness (Manskow et al., 2017), coping strategies (Baker et al., 2017; Chwalisz, 1992; Davis et al., 2009; Katz, Kravetz, \& Grynbaum, 2005), perceived social support (Baker et al., 2017; Davis et al., 2009; Ergh et al., 2003), and resilience (Simpson \& Jones, 2013). The impact of the emotional, cognitive, and behavioural functioning of the person with injury on the well-being of family members also requires examination in the mild TBI population (Ergh, Rapport, Coleman, \& Hanks, 2002). It is also important to acknowledge that our sample is not representative of all caregivers of adults with mild TBI.

In terms of future research, longer-term studies of family members in mild TBI samples may be helpful to determine patterns of health and well-being beyond the first year after injury. This will be especially important given that most physical recovery from TBIrelated injuries will have occurred by this time, but more persistent behavioural and social changes may be emerging. Finally, including similar numbers of male and female family members will help better understand the role of sex in outcomes over time, as will the use of measures with sex-based population norms.

With regards to policy and practice, several recommendations can be drawn from study findings and should be considered for inclusion in TBI guidelines. It may be helpful for clinicians and family members alike to be aware that, even within the context of mild TBI, family members may experience feelings of increased burden or stress to some 
degree. Family members may also find it helpful to be advised that any increased burden, in terms of QoL, mood or general health for example, tends to be relatively minimal and gradually resolves over the year following injury. Such a conversation and perhaps mention in existing written material provided after mild TBI, could be further supported by the provision of contact details for support organisations that may be helpful for family members should they experience any changes in their well-being. Service providers working with families impacted by mild TBI also need to be aware of and more closely monitor the well-being of high-risk family members identified in the current sample, being females, Māori and those supporting someone with a prior history of TBI

The overall picture that emerges from the current study is that family members experience improvements in well-being from 6- to 12-months. These findings suggest that family members of adults with even a mild TBI are impacted to some extent as found in moderate to severe TBI samples. By contrast though, these effects appear to diminish over the first year post-injury. Further research is needed to determine if longer-term follow-up reveals a plateau in outcomes, further improvements, or increasing difficulties over time as can be found among more severely injured samples.

\section{Financial Support}

This work was supported by the Health Research Council of NZ (HRC grant numbers 09/063A, 13/408). 


\section{Conflict of Interest}

Kelly Jones has no conflicts of interest to disclose. Nicola Starkey has no conflicts of interest to disclose. Alice Theadom has no conflicts of interest to disclose. Philip Prah has no conflicts of interest to disclose. Suzanne Barker-Collo has no conflicts of interest to disclose. Shanthi Ameratunga has no conflicts of interest to disclose. Valery Feigin has no conflicts of interest to disclose.

\section{Ethical Standards}

The authors assert that all procedures contributing to this work comply with the ethical standards of the relevant national and institutional committees on human experimentation and with the Helsinki Declaration of 1975, as revised in 2008.

\section{Acknowledgements}

We thank all caregivers who shared their experiences with us. Thank you also to the research team for their dedication and performance. A list of members of the BIONIC team is available at www.nisan.aut.ac.nz. Alice Theadom was co-funded by ABI Rehabilitation Management. This work was supported by the Health Research Council (HRC) of NZ (grants 09/063A and 13/408).

The steering committee members of the BIONIC and BIONIC4you study group are: NZ - V Feigin (chair and principal investigator), S Barker-Collo, K McPherson, A Theadom, K Jones, A Jones, B Te Ao (University of Auckland); R Kydd, P Alan Barber, V Parag, S Ameratunga (University of Auckland); N Starkey (Waikato University); A Dowell (Otago University); M Kahan, G Christey (Waikato District 
Health Board); N Hardaker (Accident Compensation Corporation), USA - P Brown (University of California). 


\section{References}

Aitken, M. E., Mele, N., \& Barrett, K. W. (2004). Recovery of injured children: parent perspectives on family needs. Archives of Physical Medicine and Rehabilitation, 85(4), 567-573. doi:http://dx.doi.org/10.1016/j.apmr.2003.06.018

Anderson, M., Simpson, G., \& Daher, M. (2017). A predictive model of resilience among family caregivers supporting relatives with traumatic brain injury (TBI) in Australia: A structural equation modelling approach. Brain Injury, 31(6-7), 815-816. doi:http://dx.doi.org/10.1080/02699052.2017.1312145

Anderson, M. I., \& Simpson, G. K. (2013). The impact of neurobehavioral impairment on family functioning and psychological well-being of male versus female caregivers of relatives with severe traumatic brain injury: Multigroup analysis. Journal of Head Trauma Rehabilitation, 28(6), 453-463.

Arango-Lasprilla, J. C., Quijano, M. C., Aponte, M., Cuervo, M. T., Nicholls, E., Rogers, H. L., et al. (2010). Family needs in caregivers of individuals with traumatic brain injury from Colombia, South America. Brain Injury, 24(7-8), 1017-1026. doi:https://doi.org/10.3109/02699052.2010.490516

Armstrong, K., \& Kerns, K. A. (2002). The assessment of parent needs following paediatric traumatic brain injury. Pediatric Rehabilitation, 5(3), 149-160. doi:http://dx.doi/org/10.1080/1363849021000039353

Australian Bureau of Statistics. (1997). 1995 National Health Survey SF-36 population norms. Canberra: ABS

Bakas, T., Champion, V., Perkins, S. M., Farran, C. J., \& Williams, L. S. (2006). Psychometric testing of the revised 15-item Bakas Caregiving Outcomes Scale. Nursing Research, 55(5), 346-355. 
Baker, A., Barker, S., Sampson, A., \& Martin, C. (2017). Caregiver outcomes and interventions: a systematic scoping review of the traumatic brain injury and spinal cord literature. Clinical Rehabilitation, 31(1), 45-60.

Barker-Collo, S. L., Wilde, N. J., \& Feigin, V. L. (2009). Trends in head injury incidence in New Zealand: A hospital-based study from 1997/1998 to 2003/2004. Neuroepidemiology, 32(1), 32-39. doi:http://dx.doi.org/10.1159/000170090

Barnfied, T. V., \& Leathem, J. M. (1998). Incidence and outcomes of traumatic brain injury and substance abuse in New Zealand prison population. Brain Injury, $12(6), 455-466$.

Bayen, E., Pradat-Diehl, P., Jourdan, C., Ghout, I., Bosserelle, V., Azerad, S., et al. (2013). Predictors of informal care burden 1 year after a severe traumatic brain injury: Results from the PariS-TBI study. Journal of Head Trauma Rehabilitation, 28(6), 408-418. doi:http://dx.doi.org/10.1097/HTR.0b013e31825413cf

Berglund, E., Lytsy, P., \& Westerling, R. (2015). Health and wellbeing in informal caregivers and non-caregivers: A comparative cross-sectional study of the Swedish general population. Health Qual Life Outcomes, 13, 109. doi:http://dx.doi.org/10.1186/s12955-015-0309-2

Bhandari, N. J., Jain, T., Marolda, C., \& ZuWallack, R. L. (2013). Comprehensive pulmonary rehabilitation results in clinically meaningful improvements in anxiety and depression in patients with chronic obstructive pulmonary disease. Journal of Cardiopulmonary Rehabilitation and Prevention, 33(2), 123-127. 
Buysse, D. J., Reynolds, C. F., Monk, T. H., Berman, S. R., \& Kupfer, D. J. (1989). The Pittsburgh Sleep Quality Index (PSQI): A new instrument for psychiatric research and practice. Psychiatry Research, 28(2), 193-213.

Carroll, L. J., Cassidy, J. D., Cancelliere, C., Côté, P., Hincapie, C. A., Kristman, V. L., et al. (2014). Systematic review of the prognosis after mild traumatic brain injury in adults: cognitive, psychiatric, and mortality outcomes: results of the international collaboration on mild traumatic brain injury prognosis. Archives of Physical Medicine and Rehabilitation, 95((3 Suppl)), S152-173. doi:http://dx.doi.org/10.1016/j.apmr.2013.08.300

Carroll, L. J., Cassidy, J. D., Holm, L., Kraus, J., \& Corondo, V. G. (2004). Methodological issues and research recommendations for mild traumatic brain injury: The WHO Collaborating Centre Task Force on Mild Traumatic Brain Injury. Journal of Rehabilitation Medicine, 43 Suppl, 113-125.

Chwalisz, K. (1992). Perceived stress and caregiver burden after brain injury: a theoretical integration. Rehabil Psychol, 37, 189-203.

Dannette, M., Fergusson, D. M., \& Boden, J. M. (2008). Ethnic identity and intimate partner violenece in a New Zealand birth cohort. Social Policy Journal of New Zealand(33), 126-145.

Davis, L., Sander, A., Struchen, M., Sherer, M., Nakase-Richardson, R., \& Malec, J. (2009). Medical and psychosocial predictors of caregiver distress and perceived burden following traumatic brain injury. Journal of Head Trauma Rehabilitation, 24(3), 145-154.

Delmonico, R., Theodore, B., Sandel, E., Armstrong, M. A., \& Camica, M. (2017). Prevalence of depression and anxiety disorders following mild traumatic brain 
injury: A retrospective cohort study. Archives of Physical Medicine and Rehabilitation, 98(10), e96.

Doser, K., \& Norup, A. (2016). Caregiver burden in Danish family members of patients with severe brain injury: The chronic phase. Brain Injury, 30(3), 334-642. doi:http://dx.doi.org/10.3109/02699052.2015.1114143

Elder, H. (2012). An examination of Maori tamariki (child) and taiohi (adolescent) traumatic brain injury within a global cultural context. Australasian Psychiatry 20(1), 20-23.

Emanuelson, I., Andersson Holmkvist, E., Bjorklund, R., \& Stalhammar, D. (2003). Quality of life and post-concussion symptoms in adults after mild traumatic brain injury: a population-based study in western Sweden. Acta Neurol Scand 108(5), 332-338.

Ennis, N., Rosenbloom, B. N., Canzian, S., \& Topolovec-Vranic, J. (2013). Depression and anxiety in parent versus spouse caregivers of adult patients with traumatic brain injury: A systematic review. Neuropsychological Rehabilitation, 23(1), 118. doi:http://dx.doi.org/10.1080/09602011.2012.712871

Ergh, T. C., Hanks, R. A., Rapport, L. J., \& Coleman, R. D. (2003). Social support moderates caregiver life satisfaction following traumatic brain injury. Journal of Clinical and Experimental Neuropsychology, 25(8), 1090-1101.

Ergh, T. C., Rapport, L. J., Coleman, R. D., \& Hanks, R. A. (2002). Predictors of caregiver and family functioning following traumatic brain injury: social support moderates caregiver distress. Journal of Head Trauma Rehabilitation, 17(2), $155-174$.

EuroQol Group. (2004). Measuring self-reported population health: An international perspective based on EQ-5D 
Feigin, V. L., Theadom, A., Barker-Collo, S., Starkey, N. J., McPherson, K., Kahan, M., et al. (2013). Incidence of traumatic brain injury in New Zealand: a populationbased study. The Lancet Neurology, 12(1), 53-64. doi:https://doi.org/10.1016/S1474-4422(12)70262-4

Findler, M., Cantor, J., Haddad, L., Gordon, W., \& Ashman, T. (2001). The reliability and validity of the SF-36 health survey questionnaire for use with individuals with traumatic brain injury. Brain Injury, 15(8), 715-723.

Hanks, R. A., Rapport, L. J., \& Vangel, S. (2007). Caregiving appraisal after traumatic brain injury: the effects of functional status, coping style, social support and family functioning. NeuroRehabilitation, 22(1), 43-52.

Hasson-Ohayon, I., Goldzweig, G., Sela-Oren, T., Pizem, N., Bar-Sela, G., \& Wolf, I. (2015). Attachment style, social support and finding meaning among spouses of colorectal cancer patients: gender differences. Palliative \& Supportive Care, 13(3), 527-535. doi:http://dx.doi.org/10.1017/S1478951513000242

Hawley, C. A. (2003). Reported problems and their resolution following mild, moderate and severe traumatic brain injury amongst children and adolescents in the UK. Brain Injury, 17(2), 105-129. doi:http://dx.doi.org/10.1080/0269905021000010131

Holder, Y., Peden, M., Krug, E., Lund, J., Gururaj, G., \& Kobusingye, O. (2001). Injury Surveillance Guidelines. Geneva: World Health Organisation.

Jansen, P., Bacal, K., \& Crengle, S. (2008). He Ritenga Whakaaro: Mâori experiences of health services. Auckland, New Zealand: Auckland: Mauriora Associates

Katz, S., Kravetz, S., \& Grynbaum, F. (2005). Wives' coping flexibility, time since husbands' injury and the perceived burden of wives of men with traumatic brain 
injury. Brain Injury, 19(1), 59-66.

doi:http://dx.doi.org/10.1080/02699050410001719970

Königs, M., Engenhorst, P. J., \& Oosterlaan, J. (2016). Intelligence after traumatic brain injury: meta-analysis of outcomes and prognosis. European Journal of Neurology, 23(1), 21-29. doi:https://doi-org.ezproxy.aut.ac.nz/10.1111/ene.1271

Kratz, A. L., Sander, A. M., Brickell, T. A., Lange, R. T., \& Carlozzi, N. E. (2017). Traumatic brain injury caregivers: A qualitative analysis of spuse and parent perspectives on quality of life. Neuropsychological Rehabilitation, 27(1), 16-37.

Kreutzer, J. S., Rapport, L. J., Marwitz, J. H., Harrison-Felix, C., Hart, T., Glenn, M., et al. (2009). Caregivers' well-being after traumatic brain injury: A multicenter prospective investigation. Arch Phys Med Rehabil, 90(6), 939-946. doi:http://dx.doi.org/10.1016/j.apmr.2009.01.010

Lerdal, A., Slåtten, K., Saghaug, E., Grov, E. K., Normann, A. P., \& Lee, K. A., ... Gay, C. L. . (2016). Sleep among bereaved caregivers of patients admitted to hospice: A 1-year longitudinal pilot study. BMJ Open, 6(1), e009345.

Li, Q., \& Loke, A. Y. (2013). A spectrum of hidden morbidities among spousal caregivers for patients with cancer, and differences between the genders: A review of the literature. Eur J Oncol Nurs, 17(5), 578-587.

doi:http://dx.doi.org/10.1016/j.ejon.2013.01.007

Liu, W., Zhu, J., Liu, J., \& Guo, Q. (2015). Psychological state and needs of family member caregivers for victims of traumatic brain injury: A cross-sectional descriptive study. International Journal of Nursing Sciences, 2(3), 231-236. doi:https://doi.org/10.1016/j.ijnss.2015.07.001 
Luo, N., Johnson, J., \& Coons, S. (2010). Using instrument-defined health state transitions to estimate minimally important differences for four preference-based health-related quality of life instruments. Med. Care., 48, 365-371.

Maas, A. I., Menon, D. K., Adelson, P. D., Andel, N., Bell, M. J., Belli, A., et al. (2017). Traumatic brain injury: integrated approaches to improve prevention, clinical care, and research. The Lancet Neurology Commission, 16(12), 9871048.

Madsen, T., Erlangsen, E., \& Orlovska, S. (2018). Association between traumatic brain injury and risk of suicide. JAMA, 320(6), 580-588.

Manskow, U. S., Friborgc, O., Røed, C., Brainef, M., Damsgardb, E., \& Ankea, A. (2017). Patterns of change and stability in caregiver burden and life satisfaction from 1 to 2 years after severe traumatic brain injury: A Norwegian longitudinal study. NeuroRehabilitation, 40(2), 211-222.

Manskow, U. S., Sigurdardottir, S., Røe, C., Andelic, N., Skandsen, T., Damsgård, E., et al. (2015). Factors affecting caregiver burden one year after severe traumatic brain injury: a prospective nationwide multicenter study. Journal of Head Trauma Rehabilitation 30, 411-423.

doi:http://dx.doi.org/10.1097/HTR.0000000000000085

Marsh, N. V., Kersel, D. A., Havill, J. H., \& Sleigh, J. W. (1998). Caregiver burden at 6 months following severe traumatic brain injury. Brain Injury, 12(3), 225-138.

Marsh, N. V., Kersel, D. A., Havill, J. H., \& Sleigh, J. W. (2002). Caregiver burden during the year following severe traumatic brain injury. Journal of Clinical and Experimental Neuropsychology, 24(4), 434-447.

doi:http://dx.doi.org/10.1076/jcen.24.4.434.1030 
Martin, C. D. (2000). More than work: Race and gender differences in caregiving burden. Journal of Family Issues, 21, 986-1005.

McAllister, S., Derrett, S., Audas, R., \& Pau, C. (2012). Well-being of carers of injured individuals from a prospective cohort study in New Zealand. Australasian Epidemiologist, 19(2), 22-26.

McInnes, K., Friesen, C. L., MacKenzie, D. E., Westwood, D. A., \& Boe, S. G. (2017). Mild traumatic brain injury (mTBI) and chronic cognitive impairment: A scoping review. PLOS One, 2017

Ministry of Health. (1999). Taking the Pulse, The 1996/97 New Zealand Health Survey. Wellington, New Zealand.

Minney, M. J., Roberts, R. M., Mathias, J. L., Raftos, J., \& Kochar, A. (2019). Service and support needs following pediatric brain injury: perspectives of children with mild traumatic brain injury and their parents. Brain Injury, 33(2), 168-182. doi:http://dx.doi.org/10.1080/02699052.2018.1540794

Mollayeva, T., Thurairajah, P., Burton, K., Mollayeva, S., Shapiro, C. M., \& Colantonio, A. (2016). The Pittsburgh sleep quality index as a screening tool for sleep dysfunction in clinical and non-clinical samples: A systematic review and meta-analysis. Sleep Medicine Reviews, 25, 52-73.

Morris, K. C. (2001). Psychological distress in carers of head injured individuals: The provision of written information. Brain Injury, 15, 239-254.

Mystakidou, K., Parpa, E., Panagiotou, I., Tsilika, E., Galanos, A., \& Gouliamos, A. (2013). Caregivers' anxiety and self-efficacy in palliative care. European Journal of Cancer Care, 22(2), 188-195.

doi:http://dx.doi.org/10.1111/ecc.12012 
Nakken, N., Janssen, D. J., van Vliet, M., de Vries, G. J., Clappers-Gielen, G. A., Michels, A. J., et al. (2017). Gender differences in partners of patients with COPD and their perceptions about the patients. International Journal of Chronic Obstructive Pulmonary Disease, 12, 95-104. doi:http://dx.doi.org/10.2147/COPD.S118871

Nonterah, C. W., Jensen, B. J., Perrin, P. B., Stevens, L. F., Cabrera, T. V., JimenezMaldonado, M., et al. (2013). The influence of TBI impairments on family caregiver mental health in Mexico. Brain Injury, 27(11), 1287e1293.

Norup, A., Snipes, D. J., Siert, L., Mortensen, E. L., Perrin, P. B., \& Arango-Lasprilla, J. C. (2013). Longitudinal trajectories of health related quality of life in Danish family members of individuals with severe brain injury. Australian Journal of Rehabilitation Counselling, 19(2), 71-83.

Oakley-Brown, M. A., Wells, J. E., \& Scott, K. M. e. (2006). Te Rau Hinengaro. The New Zealand Mental Health Survey. Wellington, New Zealand: Ministry of Health.

Omachi, T. A. (2011). Measures of sleep in rheumatologic diseases: Epworth Sleepiness Scale (ESS), Functional Outcome of Sleep Questionnaire (FOSQ), Insomnia Severity Index (ISI), and Pittsburgh Sleep Quality Index (PSQI). Arthritis Care \& Research, 63(S11), S287-S296.

Parag, V., Hackett, M. L., Yapa, C., Ngaire, K., McNaughton, H., Feigin, V. L., et al. (2008). The impact of stroke on unpaid caregivers: Results from the Auckland Regional Community Stroke Study, 2002-2003. Cerebrovascular Disease, 25(6), 548-544.

Powell, J. M., Fraser, R., Brockway, J. A., Temkin, N., \& Bell, K. R. (2015). A telehealth approach to caregiver self-management following traumatic brain 
injury: A randomized controlled trial. Journal of Head Trauma Rehabilitation, 31(3), 180-190. doi:http://dx.doi.org/10.1097/HTR.0000000000000167

Powell, J. M., Wise, E. K., Brockway, J. A., Fraser, R., Temkin, N., \& Bell, K. R.

(2017). Characteristics and concerns of caregivers of adults with traumatic brain injury. Journal of Head Trauma Rehabilitation, 32(1), E33-E41.

Puhan, M. A., Frey, M., Buchi, S., \& Schunemann, H. J. (2008). The minimal important difference of the hospital anxiety and depression scale in patients with chronic obstructive pulmonary disease. Health Qual Life Outcomes, 6, 46.

Qutub, K., Lacomis, D., Albert, S. M., \& Feingold, E. (2014). Life factors affecting depression and burden in amyotrophic lateral sclerosis caregivers. Amyotroph Lateral Scler Frontotemporal Degener. 15((3-4)), 292-297. doi:http://dx.doi.org/10.3109/21678421.2014.886699

Rumpold, T., Schur, S., Amering, M., Kirchheiner, K., Masel, E. K., Watzke, H., et al. (2015). Informal caregivers of advanced-stage cancer patients: Every second is at risk for psychiatric morbidity. Support Care Cancer, 24(5), 1975-1982. doi:http://dx.doi.org/10.1007/s00520-015-2987-z

Sady, M. D., Sander, A. M., Clark, A. N., Sherer, M., Nakase-Richardson, R., \& Malec, J. F. (2010). Relationship of preinjury caregiver and family functioning to community integration in adults with traumatic brain injury. Archives of Physical Medicine and Rehabilitation, 91(10), 1542-1550.

Samsa, G., Edelman, D., Rothman, M. L., Williams, G. R., Lipscomb, J., \& Matchar, D. (1999). Determining clinically important differences in health status measures: a general approach with illustration to the Health Utilities Index Mark II. . Pharmacoeconomics, 15, 141-155. 
Sander, A. M., Maestas, K. L., Sherer, M., Malec, J. F., \& Nakase-Richardson, R. (2012). Relationship of caregiver and family functioning to participation outcomes after postacute rehabilitation for traumatic brain injury: a multicenter investigation. Archives of Physical Medicine and Rehabilitation, 93(5), 842-848.

Scholten, A. C., Haagsma, J. A., Andriessen, T. M. J. C., Vos, P. E., Steyerberg, E. W., van Beeck, E. F., et al. (2015). Health-related quality of life after mild, moderate and severe traumatic brain injury: Patterns and predictors of suboptimal functioning during the first year after injury. Injury, 46(4), 616-624.

Schrank, B., Ebert-Vogel, A., Amering, M., Masel, E. K., Neubauer, M., Watzke, H., et al. (2016). Gender differences in caregiver burden and its determinants in family members of terminally ill cancer patients. Psycho-Oncology, 25(7), 808-814. doi:https://doi-org.ezproxy.aut.ac.nz/10.1002/pon.4005

Scott, K. M., Sarfati, D., Tobias, M. I., \& Haslett, S. J. (2000). A challenge to the crosscultural validity of the SF-36 health survey: factor structure in Maori, Pacific and New Zealand European ethnic groups. Social Science \& Medicine, 21, $1655-1664$.

Scott, K. M., Tobias, M. I., \& Sarfati, D. (1999). SF-36 health survey reliability, validity and norms for New Zealand. Australian and New Zealand Journal of Public Health, 23(4), 401-406.

Sharma, N., Chakrabarti, S., \& Grover, S. (2016). Gender differences in caregiving among family - caregivers of people with mental illnesses. World Journal of Psychiatry, 6(1), 7-17. doi:http://dx.doi.org/10.5498/wjp.v6.i1.7

Simpson, G., \& Jones, K. (2013). How important is resilience among family members supporting relatives with traumatic brain injury or spinal cord injury? Clinical Rehabilitation, 27(4), 367-377. 
Statistics New Zealand. (2017). National ethnic population projections. Retrieved from http://archive.stats.govt.nz/browse_for_stats/population/estimates_and_projectio $\underline{\text { ns/MaoriPopulationEstimates_HOTPAt30Jun17.aspx }}$

Szende, A., Janssen, B., \& Cabases, J. (Eds.). (2014). Self-Reported Population Health: An International Perspective based on EQ-5D: Springer Open

Te Ao, B., Tobias, M., Ameratunga, S., McPherson, K., Theadom, A., Dowell, A., et al. (2015). Burden of Traumatic Brain Injury in New Zealand: Incidence, prevalence and disability-adjusted life years. Neuroepidemiology, 44, 255-261. doi:http://dx.doi.org/10.1159/000431043

The EuroQol Group. (1990). EuroQol-a new facility for the measurement of healthrelated quality of life. Health Policy, 16(3), 199-208.

Theadom, A., Barker-Collo, S., Feigin, V. L., Starkey, N. J., Jones, K., Jones, A., et al. (2012). The spectrum captured: A methodological approach to studying incidence and outcomes of traumatic brain injury on a population level. Neuroepidemiology, 38, 18-29. doi:10.1159/000334746

Theadom, A., Cropley, M., Parmar, P., Barker-Collo, S., Starkey, N., Jones, K., et al. (2015). Sleep difficulties one year following mild traumatic brain injury in a population-based study. Sleep Medicine, 16(8), 926-932. doi:http://dx.doi.org/10.1016/j.sleep.2015.04.013

Theadom, A., Starkey, N., Barker-Collo, S., Jones, K., Ameratunga, S., \& Feigin, V. (2018). Population-based cohort study of the impacts of mild traumatic brain injury in adults four years post-injury. PLoS ONE, 13(1), e0191655. doi:https://doi.org/10.1371/journal.pone.0191655 
Vangel, S., Rapport, L., \& Hanks, R. A. (2011). Effects of family and caregiver psychosocial functioning on outcomes in persons with traumatic brain injury. Journal of Head Trauma Rehabilitation, 26(1), 20-29.

Wadhwa, D., Burman, D., Swami, A., Rodin, G., Lo, C., \& Zimmermann, C. (2013). Quality of life and mental health in caregivers of outpatients with advanced cancer. Psycho-Oncology, 22(2), 403-410. doi:http://dx.doi.org/10.1002/pon.2104

Wang, H. M., Patrick, D. L., Edwards, T. C., Skalicky, A. M., Zeng, H. Y., \& Gu, W. W. (2012). Validation of the EQ-5D in a general population sample in urban China. Quality of Life Research, 21(1), 155-160.

Ware, J. E., Jr., \& Sherbourne, C. D. (1992). The MOS 36-Item Short-Form Health Survey (SF-36): I. Conceptual Framework and Item Selection. Medical Care, 30, 473-483.

Ware, J. E., Snow, K. K., Kosinski, M., \& Gandek, B. G. (1993). SF-36 Health Survey: Manual and interpretation guide. Boston (MA): The Health Institute

Zhang, F., Fu, S. Y., Wang, M., \& Du, J. (2009). Correlation empirical research on the quality of life of Chinese patients with acute ischemic stoke measured by EQ5D. China Journal of Pharmaceutical Economics, 6, 5-12.

Zhou, L., Jin, H., Deng, Z. H., Cheng, X. W., Li, Z., \& Hu, S. X., et al. (2012). Measuring quality of life of hospitalized patients with severe acute respiratory infection by EQ-5D instrument: An exploratory study. Practical Preventive Medicine, 19(5), 644-647.

Zigmond, A., \& Snaith, R. (1983). The hospital anxiety and depression scale. Acta Psychiatrica Scandinavica,, 67(6), 361-370. 
Table 1. Comparison of participant and non-participant characteristics.

\begin{tabular}{lccl}
\hline & Included & Excluded & \\
$\mathrm{N}(\%)$ & $\mathrm{N}(\%)$ & p-value
\end{tabular}

\section{Family member characteristics}

Age group

$\begin{array}{llll}16-29 & 19(19.2) & 19(15.7) & 0.854 \\ 30-39 & 20(20.2) & 22(18.2) & \\ 40-49 & 27(27.3) & 37(30.6) & \\ 50+ & 33(33.3) & 43(35.5) & \\ \text { Mean (SD) } & 43.6(14.4) & 43.9(14.0) & \\ \text { Relationship to participant } & & 42(34.2) & 0.642 \\ \text { Parent } & 31(31.3) & 52(42.3) & \\ \text { Partner } & 48(48.5) & 29(23.6) & \\ \text { Other (i.e. sibling, friend, adult child) } & 20(20.2) & \end{array}$

Sex

Male

$26(26.3)$

$32(26.0)$

0.967

Female

$73(73.7)$

$91(74.0)$

\section{TBI patient characteristics}

Age group

$\begin{array}{lccc}16-29 & 42(42.4) & 58(53.2) & 0.104 \\ 30-39 & 21(21.2) & 11(10.1) & \\ 40-49 & 11(11.1) & 16(14.7) \\ 50+ & 25(25.3) & 24(22.0) \\ \text { Mean (SD) } & 36.9(18.0) & 34.6(18.2)\end{array}$


Sex

Male

Female

Ethnicity

Māori

NZ European

Other

Denominator
$62(62.6)$

$66(60.6)$

$43(39.5)$

$37(37.4)$

$24(24.2)$

$70(70.7)$

$5(5.1)$

$99(100.0)$
$22(20.2)$

0.758

$82(75.2)$

$5(4.6)$

$123(100.0)$

\section{Patient MCID improvements over time}

Physical summary (SF-36)

$18(25.7)$

Mental summary (SF-36)

$20(28.6)$

Anxiety (HADS)

$12(17.1)$

Depression (HADS)

$14(20.0)$

Denominator

$70(70.7)$

\section{Injury factors}

Mechanism of injury

MVA

Fall

Mechanical object

Assault

Other

Accidental injury

Yes

No
$19(19.4)$

$35(35.7)$

$22(22.4)$

$20(20.4)$

$2(2)$
$0(0.0)$

$25(23.2) \quad 0.575$

$34(31.5)$

$26(24.1)$

$23(21.3)$ 
Recurrent TBI (within 12 months)

Yes

No

Prior TBI

Yes

No

Denominator
$13(13.1)$

$86(86.9)$

$13(11.9)$

$96(88.1)$
0.793

$53(55.2)$

$46(43.4)$

0.094

$43(44.8)$

$60(56.6)$

$99(100.0)$

$109(100.0)$

SD $=$ Standard deviation. NZ $=$ New Zealand. (-) Denotes data not available. $\mathrm{TBI}=$ Traumatic Brain Injury. SF-36 = Short Form-36 Health Survey. HADS = Hospital Anxiety and Depression scale. MVA = Motor Vehicle Accident. 
Table 2. Significant other group mean (SD) health outcomes over time and crude regression estimates.

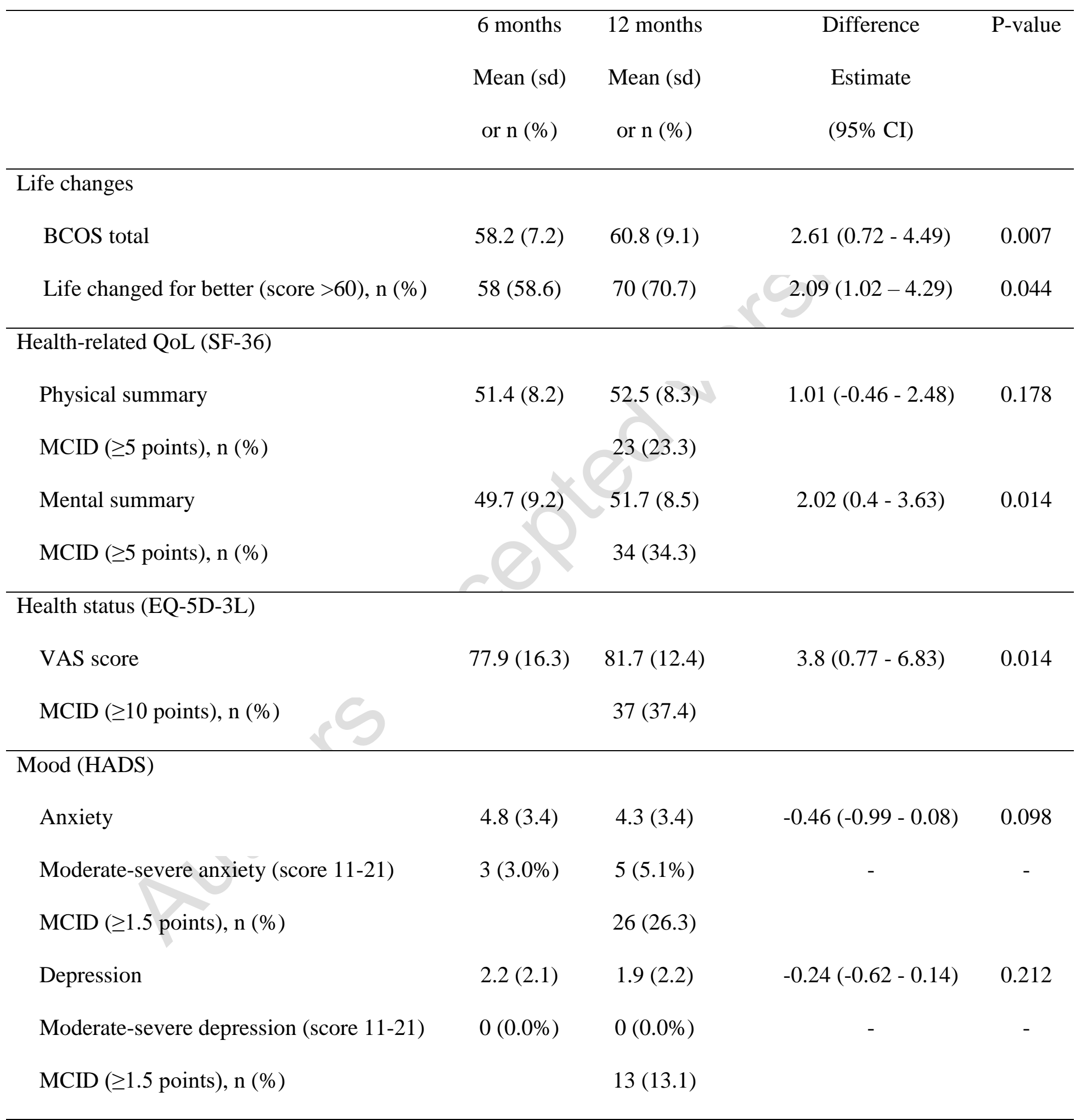

Sleep (PSQI) 
PSQI total

Poor sleep quality (PSQI score >5)

MCID ( $\geq 3$ units), n (\%)

$$
4.7(3.8)
$$

$4.3(2.8)$

$-0.41(-1.02-0.2)$

0.189

Denominator
$35(35.3 \%) \quad 25(25.8 \%)$

$0.43(0.18-1.05)$

$11(11.3)$

99

BCOS = Bakas Caregiver Outscomes Scale. SF-36 = Short Form-36 Health Survey. MCID = Minimally Clinically

Important Difference. VAS = Visual Analogue Scale. HADS = Hospital Anxiety and Depression Scale. PSQI = Pittsburgh Sleep Quality Index. Note: (-) denotes data unavailable due to too few outcomes. 
Table 3. Estimates from five significant mixed models where overall change over time differs by family member or patient characteristics.

\section{Estimate $95 \%$ Confidence interval $\quad \mathrm{P}$-value}

\section{Physical summary (SF36)}

Male intercept

51.15

48.04 to 54.26

$<0.001$

Male slope

$-1.99$

$-4.78-0.80$

0.162

Female intercept

51.54

$49.68-53.4$

$<0.001$

Female slope

2.08

$0.42-3.74$

0.014

\section{Bodily pain (SF36)}

Male intercept

77.65

$69.2-86.10$

$<0.001$

Male slope

$-5.58$

$-14.84-3.68$

0.238

Female intercept

78.77

$73.72-83.81$

$<0.001$

Female slope

7.22

$1.69-12.75$

0.010

\section{Mental summary (SF36)}

TBI patient Māori intercept

49.15

$45.66-52.64$

$<0.001$

TBI patient Māori slope

$-0.73$

$-3.94-2.48$

0.655

TBI patient European intercept

49.54

$47.5-51.58$

$<0.001$

TBI patient European slope

$1.19-4.95$

$<0.001$

TBI patient Other intercept

53.78

$46.14-61.42$

$<0.001$

TBI patient Other slope

0.37

$-6.66-7.41$

0.917 
Total life changes (BCOS)

TBI patient Male intercept

TBI patient Male slope

TBI patient Female intercept

TBI patient Female slope
57.11

4.34

60.00

$-0.30$
$2.00-3.26$

$-1.34--0.24$

$1.34-2.47$

$-0.4-0.59$
$<0.001$

$<0.001$

$<0.001$

0.846

\section{Depression (HADS)}

TBI patient No prior TBI intercept

$-0.79$

1.91

0.09

TBI patient Prior TBI slope 